\title{
Investigation of Cumulative Retrospective Cost Adaptive Control for Missile Application
}

\author{
Robert Fuentes, Jesse B. Hoagg, Blake J. Anderton, Anthony M. D’Amato, Dennis S. Bernstein* ${ }^{\dagger}$
}

\begin{abstract}
A cumulative retrospective cost adaptive control algorithm is augmented to a gain-scheduled three loop autopilot to assess performance in a nonlinear three-degree-of-freedom missile model. The cumulative retrospective cost adaptive controller requires minimal model information; specifically estimates of the relative degree, 1st nonzero Markov parameter, and nonminimum phase zeros of the linearized missile model. A rate gyro sensor failure is applied to the model and the performance of the control techniques is investigated through an analysis of air-to-air intercept scenarios. The results indicate that an adaptive controller can, to some degree, improve the missile's ability to engage a target when such a failure occurs.
\end{abstract}

\section{Nomenclature}

$m \quad$ Missile mass

$I_{y y} \quad$ Missile Inertia

$g \quad$ Acceleration due to gravity

$X, Z$ Inertial position in the $\mathrm{X}-\mathrm{Z}$ plane

$U \quad$ Inertial velocity component along the body frame $\mathrm{x}$-axis of the center of gravity $(\mathrm{CG})$

$W \quad$ Inertial velocity component along the body frame $\mathrm{z}$-axis of the $\mathrm{CG}$

$V_{m} \quad$ Magnitude of missile velocity

$M \quad$ Mach number

$A_{z, \mathrm{P}}$ Acceleration component along body frame $\mathrm{z}$-axis at point $P$ along the body $\mathrm{x}$-axis

$\alpha \quad$ Angle of attack

$\theta \quad$ Pitch angle

$q \quad$ Body rate

$\rho \quad$ Air density

$S_{\text {ref }}$ Reference area

$d_{\text {ref }}$ Reference length

$C_{x} \quad$ Aerodynamic force coefficient along the body frame x-axis

$C_{z} \quad$ Aerodynamic force coefficient along the body frame z-axis

$C_{m} \quad$ Aerodynamic moment coefficient along the body y-axis at the CG

$\bar{q} \quad$ Dynamic pressure

$\delta_{p} \quad$ Tail fin angle

$T \quad$ Thrust along body frame $\mathrm{x}$-axis

\section{Introduction}

Application of adaptive control to an agile tail-controlled missile system is a difficult problem. First, such missile systems typically have an initial wrong-way response in measured acceleration due to the presence of a nonminimumphase zero ${ }^{1}$ in the linearization about trim. Therefore, standard adaptive control techniques designed for minimumphase systems ${ }^{2,3}$ are not well-suited for missile control. In fact, adaptive controllers based on a minimum phase assumption can fail to produce stability throughout the range of tactical operation. Second, actuator delays, aerodynamic effects, and structural flexibility introduce significant complexity to the problem. Third, agile tail-controlled missiles require fast response times in order to achieve acceleration commands generated by guidance control laws. Finally, many assumptions made when designing an adaptive controller for continuous time systems often fail to hold when the (missile) system is sampled and controlled using zero-order-hold inputs.

Several studies of adaptive control applied to the missile problem are available in the literature ${ }^{4-11}$. More specifically, neural network adaptive control with integral action is currently being implemented on the Joint Direct Attack Munition (JDAM) ${ }^{4}$. Adaptive control applied to bank-to-turn missiles has been investigated using neural networks ${ }^{5-8}$. Variants of $\mathcal{L}_{1}$ adaptive control show improved performance can be achieved when applied to a linear, tail-controlled

${ }^{*}$ R. Fuentes and B. Anderton are with Raytheon Missile Systems in Tucson, AZ.

$\dagger$ J.B. Hoagg, A.M. D’Amato, and D.S. Bernstein are with the Department of Aerospace Engineering, University of Michigan 
missile model ${ }^{9,10}$. Model reference adaptive control using a retrospective correction filter shows that a given linear, tail-controlled missile model could adapt to changes in fin control effectiveness ${ }^{11}$.

Discrete-time adaptive controllers using a retrospective cost have been shown to be effective for minimum phase and nonminimum phase systems ${ }^{12-14}$. A stability and convergence proof is available for the minimum-phase case ${ }^{15}$. The fundamental concept behind retrospective cost adaptive control is the creation of a retrospective performance variable, which adjusts the performance measurement based on the difference between the actual past control inputs and the recomputed past control inputs, assuming that the current controller had been used in the past. A retrospective cost function is defined which incorporates this variable and this cost function is optimized to determine the adaptive update law. In instantaneous retrospective cost adaptive control, the adaptive law optimizes a cost function, which depends on the retrospective performance at the current time step ${ }^{12,13}$. In contrast, cumulative retrospective cost adaptive control minimizes a cumulative retrospective cost function, which is a function of the retrospective performance at the current time step and all previous time steps ${ }^{14}$. The cumulative retrospective cost algorithm has shown to converge quickly for regulation of nonminimum-phase systems, with the caveat being that information about the nonminimum-phase zeros is incorporated in the design. The discrete-time adaptive algorithm is also well-suited for implementation on a processor. Thus, system delays can be approximated by the shift operator and easily incorporated into the algorithm.

Motivated by the promising performance of retrospective cost adaptive control ${ }^{11}$, in this paper we use a cumulative retrospective cost adaptive controller ${ }^{14}$ to control an open source Mathworks 3 DOF tail-controlled nonlinear missile model. The baseline missile control system uses a gain-scheduled three loop autopilot topology ${ }^{16-18}$. In the adaptive control design, we augment the cumulative retrospective cost adaptive algorithm to the missile autopilot. Both systems are tested using a rate gyro failure that introduces significant gyro sensor noise into the closed loop system.

The paper is organized as follows. Section II provides the aerodynamic equations of motion, discusses details behind the nonlinear missile model, and outlines the adaptive augmentation strategy. The retrospective cost adaptive control algorithm is reviewed in section III. Section IV shows a single engagement scenario where the adaptive controller reduces miss distance significantly when the rate gyro failure occurs. Finally, the more stringent monte carlo analysis presented in section V illustrates the RCAC controller yields a modest overall reduction in miss distance.

\section{Nonlinear Missile Model and Three Loop Autopilot}

Consider the nonlinear three-degree-of-freedom missile model,

$$
\begin{aligned}
\dot{X} & =U \cos \theta+W \sin \theta \\
\dot{Z} & =-U \sin \theta+W \cos \theta \\
\dot{\theta} & =q \\
\dot{U} & =\frac{1}{m} T+\frac{\bar{q} S_{\mathrm{ref}}}{m} C_{x}(\alpha, M)-q W-g \sin \theta \\
\dot{W} & =\frac{\bar{q} S_{\mathrm{ref}}}{m} C_{z}\left(\alpha, M, \delta_{p}\right)+q U+g \cos \theta \\
\dot{q} & =\frac{\bar{q} S_{\mathrm{ref}} d_{\mathrm{ref}}}{I_{y y}} C_{m}\left(\alpha, M, \delta_{p}, q\right)
\end{aligned}
$$

where the dynamic pressure is given by $\bar{q}=1 / 2 \rho V_{m}^{2}$ and angle of attack is defined as $\alpha=\operatorname{atan}(W / U)$. Measurements of the body rate $\dot{q}$ and lateral missile acceleration are assumed to take place at the inertial measurement unit (IMU) location. The lateral acceleration at the IMU is related to that measured at the center of gravity through the equation

$$
A_{z, \mathrm{IMU}}=A_{z, \mathrm{CG}}-\dot{q} x_{\mathrm{IMU}},
$$

where $x_{I M U}$ is the distance from the CG to IMU. Here we assume that the missile IMU is forward of the CG location. The nonlinear model in equations (1)-(7) is linearized about trim points in the region of operation for the purpose of control design. Additionally, a second order fin actuator model of the form

$$
u_{p}(s)=\frac{\omega_{a}^{2}}{s^{2}+2 \zeta_{a} \omega_{a} s+\omega_{a}^{2}} u_{a}(s),
$$

is used, where $u_{a}$ is the fin actuator command and $\omega_{a}, \zeta_{a}$ represent the natural frequency and damping ratio, respectively, of the actuator model. A gain-scheduled three loop autopilot ${ }^{16}$ is designed for each trim point. The continuous time representation for this control design is

$$
\tilde{u}(s)=K_{q} q(s)+\frac{1}{s}\left(K_{\theta} q(s)+K_{I}\left(K_{s s} A_{z, \mathrm{cmd}}-A_{z, \mathrm{IMU}}\right)\right),
$$

where $K_{q}, K_{\theta}, K_{I}, K_{s s}$ are gain-scheduled control gains and $A_{z, \mathrm{cmd}}$ is the lateral acceleration command for the IMU location provided by the missile's guidance system. A proportional navigation guidance law generates the acceleration 
command, while a target seeker model provides the guidance algorithm with line of sight rate and closing velocity information. The overall missile system model is illustrated in Figure 1.

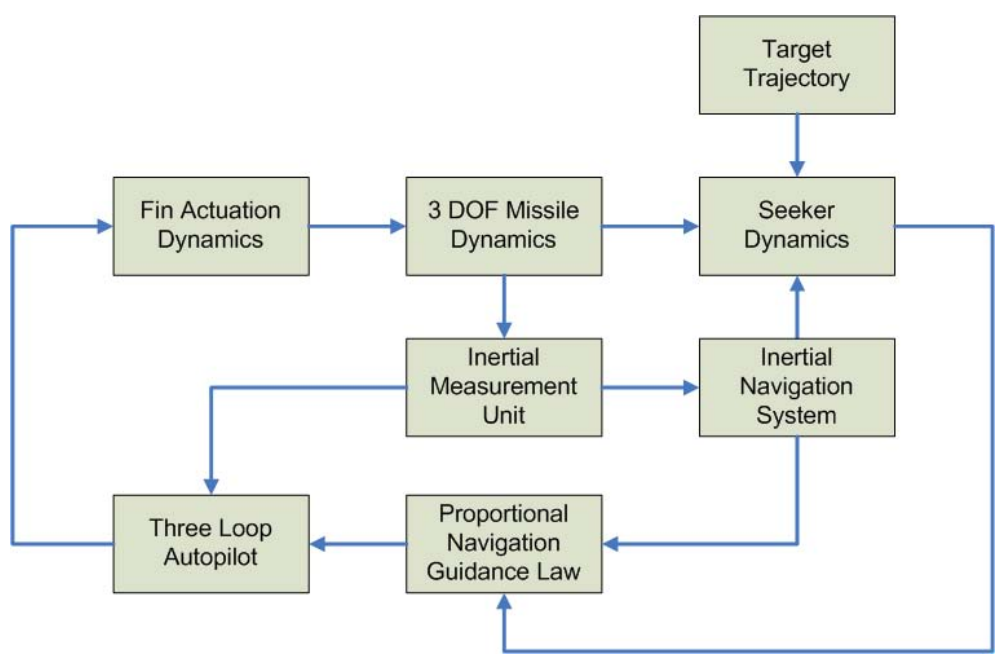

Figure 1. Missile System Block Diagram.

The trim linear model, actuator, and three loop autopilot based on equations (1)-(9) can be discretized to create a system of the form

$$
\begin{aligned}
x(k+1) & =A x(k)+B u(k), \\
y(k) & =C x(k)+D_{2} w(k), \\
z(k) & =E_{1} x(k)+E_{0} w(k),
\end{aligned}
$$

where $x(k) \in \mathbb{R}^{n}$ is a state vector, $y(k) \in \mathbb{R}^{l_{y}}$ is a vector of measurements, $z(k) \in \mathbb{R}^{l_{z}}$ consists of performance variables, $u(k) \in \mathbb{R}^{l_{u}}$ is the control input, $w(k) \in \mathbb{R}^{l_{w}}$ is an exogenous input vector consisting of reference input commands and disturbances, and $k \geq 0$.

In this application, the adaptive controller produces a fin actuator command $u(k)$ that minimizes the acceleration error performance variable

$$
z(k)=A_{z, \mathrm{IMU}}(k)-A_{z, \mathrm{cmd}}(k) .
$$

The adaptive control input augments the three loop autopilot such that

$$
u_{a}(k)=u(k)+\tilde{u}(k) .
$$

Measurement vector components can be constructed from combinations of available IMU measurements, acceleration performance variables, and measured exogenous inputs.

A time-series model from $u$ and $w$ to $z$ is obtained from (10) and (12)

$$
z(k)=\sum_{i=1}^{n}-\alpha_{i} z(k-i)+\sum_{i=d}^{n} \beta_{i} u(k-i)+\sum_{i=0}^{n} \gamma_{i} w(k-i),
$$

where $\alpha_{1}, \ldots, \alpha_{n} \in \mathbb{R}, \beta_{d}, \ldots, \beta_{n} \in \mathbb{R}^{l_{z} \times l_{u}}, \gamma_{0}, \ldots, \gamma_{n} \in \mathbb{R}^{l_{z} \times l_{w}}$, and the relative degree $d$ is the smallest nonnegative integer $i$ such that the $i$ th Markov parameter is nonzero. Since $i>0$, the Markov parameter sequence is given by $H_{i} \equiv E_{1} A^{i-1} B$. Furthermore, $H_{d}=\beta_{d}$.

\section{Overview of the Cumulative Retrospective Cost Adaptive Controller}

In this section, we review the cumulative retrospective cost adaptive control algorithm for the discrete-time linear model represented by equations (10)-(12). We use a strictly proper time-series controller of order $n_{\mathrm{c}}$, such that the control $u(k)$ is given by

$$
u(k)=\sum_{i=1}^{n_{\mathrm{c}}} M_{i}(k) u(k-i)+\sum_{i=1}^{n_{\mathrm{c}}} N_{i}(k) y(k-i),
$$


where, for all $i=1, \ldots, n_{\mathrm{c}}, M_{i} \in \mathbb{R}^{l_{u} \times l_{u}}$ and $N_{i} \in \mathbb{R}^{l_{u} \times l_{y}}$ are determined by the adaptive control law presented below. The control (16) can be expressed as

$$
u(k)=\vartheta(k) \phi(k)
$$

where

$$
\vartheta(k) \equiv\left[\begin{array}{llllll}
N_{1}(k) & \cdots & N_{n_{\mathrm{c}}}(k) & M_{1}(k) & \cdots & M_{n_{\mathrm{c}}}(k)
\end{array}\right]
$$

and

$$
\phi(k) \equiv\left[\begin{array}{c}
y(k-1) \\
\vdots \\
y\left(k-n_{\mathrm{c}}\right) \\
u(k-1) \\
\vdots \\
u\left(k-n_{\mathrm{c}}\right)
\end{array}\right] \in \mathbb{R}^{n_{\mathrm{c}}\left(l_{u}+l_{y}\right)} .
$$

Next, we define the retrospective performance

$$
\hat{z}(\hat{\vartheta}, k) \equiv z(k)+\sum_{i=d}^{\nu} \bar{\beta}_{i}[\hat{\vartheta}-\vartheta(k-i)] \phi(k-i),
$$

where $\nu \geq d, \hat{\vartheta} \in \mathbb{R}^{l_{u} \times\left(n_{c}\left(l_{y}+l_{u}\right)\right)}$ is an optimization variable used to derive the adaptive law, and $\bar{\beta}_{d}, \ldots, \bar{\beta}_{\nu} \in \mathbb{R}^{l_{z} \times l_{u}}$ are estimates of the Markov parameters $H_{d}, \ldots, H_{\nu}$. Other choices of $\bar{\beta}_{d}, \ldots, \bar{\beta}_{\nu}$ are available ${ }^{14}$.

Defining $\hat{\Theta} \equiv \operatorname{vec} \hat{\vartheta} \in \mathbb{R}^{n_{c} l_{u}\left(l_{y}+l_{u}\right)}$ and $\Theta(k) \equiv \operatorname{vec} \vartheta(k) \in \mathbb{R}^{n_{c} l_{u}\left(l_{y}+l_{u}\right)}$, it follows that

$$
\hat{z}(\hat{\Theta}, k)=z(k)+\sum_{i=d}^{\nu} \Phi_{i}^{\mathrm{T}}(k)[\hat{\Theta}-\Theta(k-i)]=z(k)-\sum_{i=d}^{\nu} \Phi_{i}^{\mathrm{T}}(k) \Theta(k-i)+\Psi^{\mathrm{T}}(k) \hat{\Theta},
$$

where, for $i=d, \ldots, \nu$,

$$
\Phi_{i}(k) \equiv \phi(k-i) \otimes \bar{\beta}_{i}^{\mathrm{T}} \in \mathbb{R}^{\left(n_{c} l_{u}\left(l_{y}+l_{u}\right)\right) \times l_{z}},
$$

where $\otimes$ represents the Kronecker product, and

$$
\Psi(k) \equiv \sum_{i=d}^{\nu} \Phi_{i}(k) .
$$

Now, define the cumulative retrospective cost function

$$
J(\hat{\Theta}, k) \equiv \sum_{i=0}^{k} \lambda^{k-i} \hat{z}^{\mathrm{T}}(\hat{\Theta}, i) R \hat{z}(\hat{\Theta}, i)+[\hat{\Theta}-\Theta(0)]^{\mathrm{T}} Q[\hat{\Theta}-\Theta(0)],
$$

where $\lambda \in(0,1]$, and $R \in \mathbb{R}^{l_{z} \times l_{z}}$ and $Q \in \mathbb{R}^{\left(n_{c} l_{u}\left(l_{y}+l_{u}\right)\right) \times\left(n_{c} l_{u}\left(l_{y}+l_{u}\right)\right)}$ are positive definite. Note that $\lambda$ serves as a forgetting factor, which allows more recent data to be weighted more heavily than past data.

The cumulative retrospective cost function (24) is minimized by a recursive least-squares (RLS) algorithm with a forgetting factor ${ }^{19-21}$. Therefore, $J(\hat{\Theta}, k)$ is minimized by the adaptive law

$$
\begin{aligned}
& \Theta(k+1)=\Theta(k)-P(k) \Psi(k)\left[\lambda R^{-1}+\Psi^{\mathrm{T}}(k) P(k) \Psi(k)\right]^{-1} z_{\mathrm{R}}(k), \\
& P(k+1)=\frac{1}{\lambda} P(k)-\frac{1}{\lambda} P(k) \Psi(k)\left[\lambda R^{-1}+\Psi^{\mathrm{T}}(k) P(k) \Psi(k)\right]^{-1} \Psi^{\mathrm{T}}(k) P(k),
\end{aligned}
$$

where $P(0)=Q^{-1}, \Theta(0) \in \mathbb{R}^{n_{c} l_{u}\left(l_{y}+l_{u}\right)}$, and the retrospective performance measurement $z_{\mathrm{R}}(k) \equiv \hat{z}(\Theta(k), k)$. Note that $z_{\mathrm{R}}(k)$ is computable from (21) using measured signals $z, y, u, \vartheta$, and the matrix coefficients $\bar{\beta}_{d}, \ldots, \bar{\beta}_{\nu}$. The cumulative retrospective cost adaptive control law is thus given by (25), (26), and

$$
u(k)=\vartheta(k) \phi(k)=\operatorname{vec}^{-1}(\Theta(k)) \phi(k) .
$$

The key feature of the adaptive control algorithm is the use of the retrospective performance (21), which modifies the performance variable $z(k)$ based on the difference between the actual past control inputs $u(k-d), \ldots, u(k-\nu)$ and the recomputed past control inputs $\hat{u}(\hat{\Theta}, k-d) \equiv \operatorname{vec}^{-1}(\hat{\Theta}) \phi(k-d), \ldots, \hat{u}(\hat{\Theta}, k-\nu) \equiv \operatorname{vec}^{-1}(\hat{\Theta}) \phi(k-\nu)$, assuming that the current controller $\hat{\Theta}$ had been used in the past. 


\section{Individual Scenario Results}

We investigate four air-to-air missile intercept scenarios with interceptor initially traveling horizontal at Mach 3.0 and target velocity horizontal at a constant Mach 1.0 at an altitude 500 meters above the initial interceptor position. The feedback variable is chosen as $y(k)=z(k)$, hence the missile plant model yields $l_{u}=l_{y}=1$. By defining the exogenous signal vector to consist of the guidance acceleration command $A_{z, \mathrm{cmd}}$ and the gyro sensor noise, we have $l_{w}=2$. A cumulative retrospective cost adaptive controller of order 25 is used, the RLS covariance initialization is set to $P(0)=10^{-5} I$, and the first 10 Markov parameters of the linearized model are gain scheduled in the design for each trim point.

In the nominal, no failure scenario, the three loop autopilot is used without the adaptive controller. During the first 0.8 seconds, the seeker searches for and acquires the target. The proportional navigation acceleration command is tracked by the three loop autopilot during the remainder of the engagement. Figure 2 illustrates the maximum lateral acceleration reached is $35 \mathrm{~g}$ with the angle of attack reaching no more than 16 degrees. The missile achieves a final miss distance of 0.3 meter with trajectory given in Figure 3 . In the second scenario, we augment the three loop autopilot with the adaptive controller. Noticeable changes are produced in the system behavior that can be seen in Figure 2. However, Figure 5 shows that the trajectory is not altered significantly and the augmented adaptive system produces a comparable miss distance of 0.2 meter.

For the failure scenarios each assume that Gaussian zero-mean white noise with standard deviation $0.1 \mathrm{rad} / \mathrm{sec}$ corrupts the rate gyro measurement. The noise not only interferes with the operation of the autopilot, but the guidance system is also directly affected. In the non-adaptive failure scenario, oscillatory motions appear in the system throughout the engagement as is shown in Figure 6. The trajectory is illustrated in Figure 7, and the non-adaptive controller results in a miss distance of approximately 27 meters.

The rate gyro failure also impacts performance in the adaptive case, the oscillatory motions become more pronounced in Figure 8 and the augmented adaptive controller saturates the fin commands in an attempt to minimize the error between measured and commanded acceleration. However, the trajectory shown in Figure 9 indicates that missile meets the overall control objective with a miss distance of 0.9 meter.

\section{Design of Experiment Results}

A design of experiments study was developed to assess the overall performance impact of RCAC algorithm designs on intercept miss distance when the rate gyro noise in the previous section is applied. The adaptive control design variables and cases chosen for this study are provided in Table 1. The monte carlo variables, distribution functions, and ranges are provided in Table 2.

For each individual case in Table 1, we investigate the influence of the design variables on miss distance to determine a good candidate adaptive control design. Figure 10 compares miss distance results in the no gyro noise case for each controller input combination. All combinations produce similar mean miss distances within a meter. When the rate gyro noise failure is applied, Figure 11 shows miss distance degrades in all cases. However, the median miss distance when the performance variable $z$ is a component of $y$ is reduced by over 7 meters for the combined set of adaptive controller orders. When controller order is investigated, Figures 12 and 13 indicate the design variable has a lesser influence on miss distance. Thus, a good candidate for a controller design would be of low order to gain computational efficiency. Based on these results, a reasonable RCAC design would include the performance variable and gyro rate as measurement inputs with controller order 10. Figure 14 indicates median miss distance is reduced by roughly 9 meters when compared with the non-adaptive case in Figure 11.

Table 1. Design Variables

$\begin{array}{cccc}\text { Variable } & \text { Controller Order } n_{\mathrm{c}} & \text { Measurement Input } y & \text { Gyro Noise Failure } \\ \text { Range } & {[10,15,20]} & z \text { only, } q \text { only, both } z \text { and } q & \text { on or off }\end{array}$

\section{Conclusion}

Through a design of experiments analysis, we have shown that a cumulative retrospective cost adaptive controller can produce a modest improvement in interceptor miss distance when a significant increase in rate gyro noise occurs. Although tuning an adaptive controller for a specific scenario can produce drastic improvements in miss distance, such single point analyzes often lead to overly optimistic performance predictions. Finally, we show that the design of experiments process can be used to find good adaptive controller design candidates. 
Table 2. Monte Carlo Variables

$\begin{array}{ccc}\text { Variable } & \text { Distribution Function } & \text { Range } \\ \text { Initial Range to Target } & \text { Uniform } & 3 \text { to } 7 \mathrm{~km} \\ \text { Initial Missile Elevation } & \text { Uniform } & 2.1 \text { to } 4.4 \mathrm{~km} \\ \text { Initial Relative Elevation Angle } & \text { Uniform } & -3 \text { to }+3 \mathrm{deg} \\ \text { Initial Missile Mach } & \text { Uniform } & 2.5 \text { to } 3.5 \\ \text { Initial Angle of Attack } & \text { Uniform } & 0 \text { to } 1.3 \mathrm{deg} \\ \text { Initial Target Trajectory Angle } & \text { Normal } & \text { Mean } 180 \mathrm{deg} \text { with } 3 \sigma=10 \mathrm{deg} \\ \text { Initial Moment of Inertia } & \text { Normal } & \text { Mean } 247 \mathrm{~kg} \mathrm{~m}^{2} w i t h \sigma=5 \% \\ C_{x}, C_{z}, C_{m} & \text { Normal } & \text { Nominal coefficient values with } \sigma= \pm 15 \%\end{array}$

\section{References}

${ }^{1}$ Hoagg, J. and Bernstein, D., "Nonminimum-Phase Zeros - much to do about nothing - classical control revisted part II," IEEE Contr. Sys. Mag., Vol. 27, No. 3, 2007, pp. 45-57.

${ }^{2}$ Narendra, K. and Annaswamy, A., Stable Adaptive Systems, Prentice-Hall, 1989.

${ }^{3}$ Åström, K. and Wittenmark, B., Adaptive Control, Addison-Wesley, 1995.

${ }^{4}$ Sharma, M., Lavretsky, E., and Wise, K., "Application and Flight Testing of an Adaptive Autopilot on Precision Guided Munitions," Proc. AIAA Guid., Nav. Contr. Conf., Keystone, CO, 2006, AIAA 2006-6568.

${ }^{5} \mathrm{Fu}$, L.-C., Chang, W.-D., Chuang, D.-M., Kuo, T.-S., Wang, T.-C., and Tsai, C.-W., "A Nolinlinear Constant Bearing Guidance and Adaptive Autopilot Design for BTT Missiles," Proc. Amer. Contr. Conf., Vol. 5, Albuquerque, NM, 1997, pp. 2774 - 2778.

${ }^{6}$ McDowell, D., Irwin, G., Lightbody, G., and McConnell, G., "Hybrid neural adaptive control for bank-to-turn missiles," IEEE Trans. Contr. Sys. Tech., Vol. 5, No. 3, 1997, pp. 297308.

${ }^{7} \mathrm{McF}$ arland, M. and Calise, A., "Adaptive nonlinear control of agile antiair missiles using neural networks," IEEE Trans. Contr. Sys. Tech., Vol. 8, No. 5, 2000, pp. 749756.

${ }^{8}$ Lin, C.-K. and Wang, S.-D., "An adaptive $H^{\infty}$ controller design for bank-to-turn missiles using ridge Gaussian neural networks," IEEE Trans. Neural Networks, Vol. 15, No. 6, 2004, pp. 1507-1506.

${ }^{9}$ Wang, J., Cao, C., Hovakimyan, N., Hindman, R., and Ridgely, B., “ $\mathcal{L}_{1}$ Adaptive Controller for a Missile Longitudinal Autopilot Design," Proc. AIAA Guid., Nav. Contr. Conf., Honolulu, HI, 2008.

${ }^{10}$ Cao, C. and Hovakimyan, N., " $\mathcal{L}_{1}$ Adaptive Output-Feedback Controller for Non-Strictly-Positive-Real Reference Systems: Missile Longitudinal Autopilot Design,” AIAA J. Guid. Contr. Dyn., Vol. 32, No. 3, 2009, pp. 717-726.

${ }^{11}$ Santillo, M. and Bernstein, D., "Inherent Robustness of Minimal Modeling Discrete-Time Adaptive Control to Flight Anomalies," Proc. AIAA Guid., Nav. Contr. Conf., Honolulu, HI, 2008, AIAA-2008-7289.

${ }^{12}$ Venugopal, R. and Bernstein, D. S., "Adaptive disturbance rejection using ARMARKOV/Toeplitz models," IEEE Trans. Contr. Sys. Tech., Vol. 8, No. 2, 2000, pp. 257-269.

${ }^{13}$ Santillo, M. and Bernstein, D., "A Retrospective Correction Filter for Discrete-Time Adaptive Control of Nonminimum-Phase Systems," Proc. IEEE Conf. Dec. Contr., Cancun, MX, 2008, pp. 690-695.

${ }^{14}$ Hoagg, J. and Bernstein, D., "Cumulative Retrospective Cost Adaptive Control with RLS-Based Optimization," Proc. Amer. Contr. Conf., Baltimore, MD, 2010, Accepted.

${ }^{15}$ Hoagg, J. and Bernstein, D., "Discrete-time adaptive command following and disturbance rejection with unknown exogenous dynamics," IEEE Trans. Autom. Contr., Vol. 53, No. 4, 2008, pp. 912-928.

${ }^{16}$ Zarchan, P., Tactical and Strategic Missile Guidance, AIAA, 5th ed., 2007.

${ }^{17}$ Mracek, C. and Ridgely, D., "Missile Longitudinal Autopilots: Connections between Optimal Control and Classical Topologies," Proc. AIAA Guid., Nav. Contr. Conf., San Francisco, CA, 2005, AIAA 2005-6381.

${ }^{18}$ Mracek, C. and Ridgely, D., "Missile Longitudinal Autopilots: Comparison of Multiple Three Loop Topologies," Proc. AIAA Guid., Nav. Contr. Conf., San Francisco, CA, 2005, AIAA 2005-6380.

${ }^{19}$ Goodwin, G. C. and Sin, K. S., Adaptive Filtering, Prediction, and Control, Prentice Hall, 1984.

${ }^{20}$ Åström, K. J. and Wittenmark, B., Adaptive Control, Addison-Wesley, 2nd ed., 1995.

${ }^{21}$ Tao, G., Adaptive Control Design and Analysis, Wiley, 2003. 

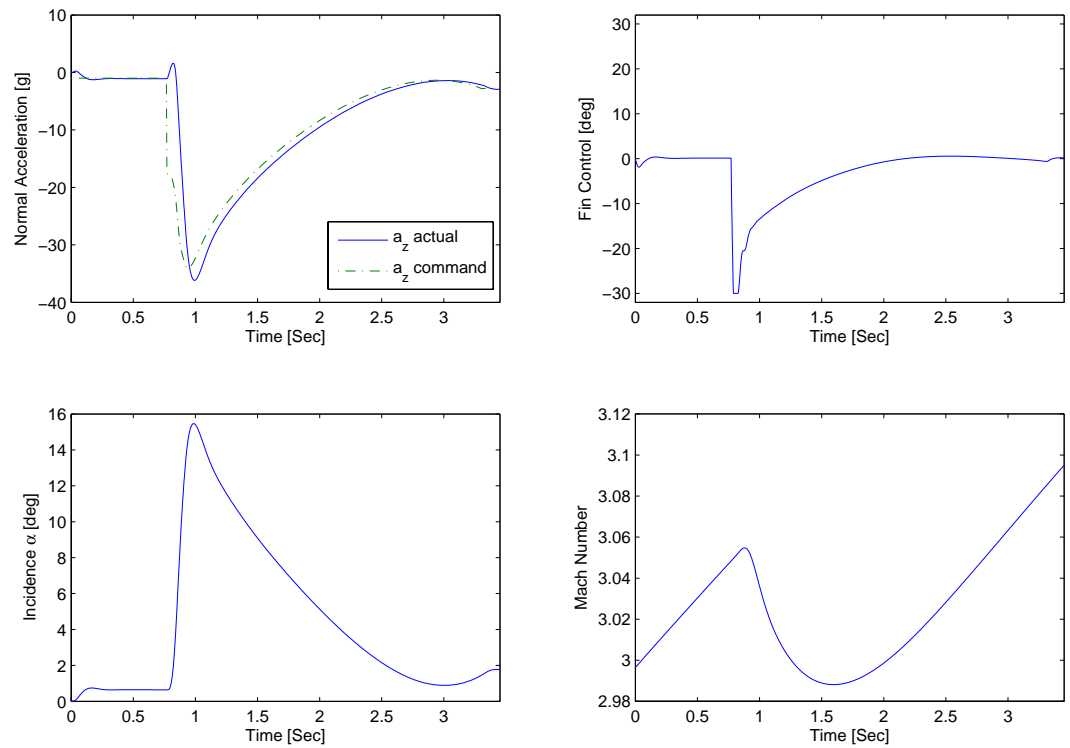

Figure 2. Time histories of measurements and control inputs for the nominal engagement scenario, no adaptive case.

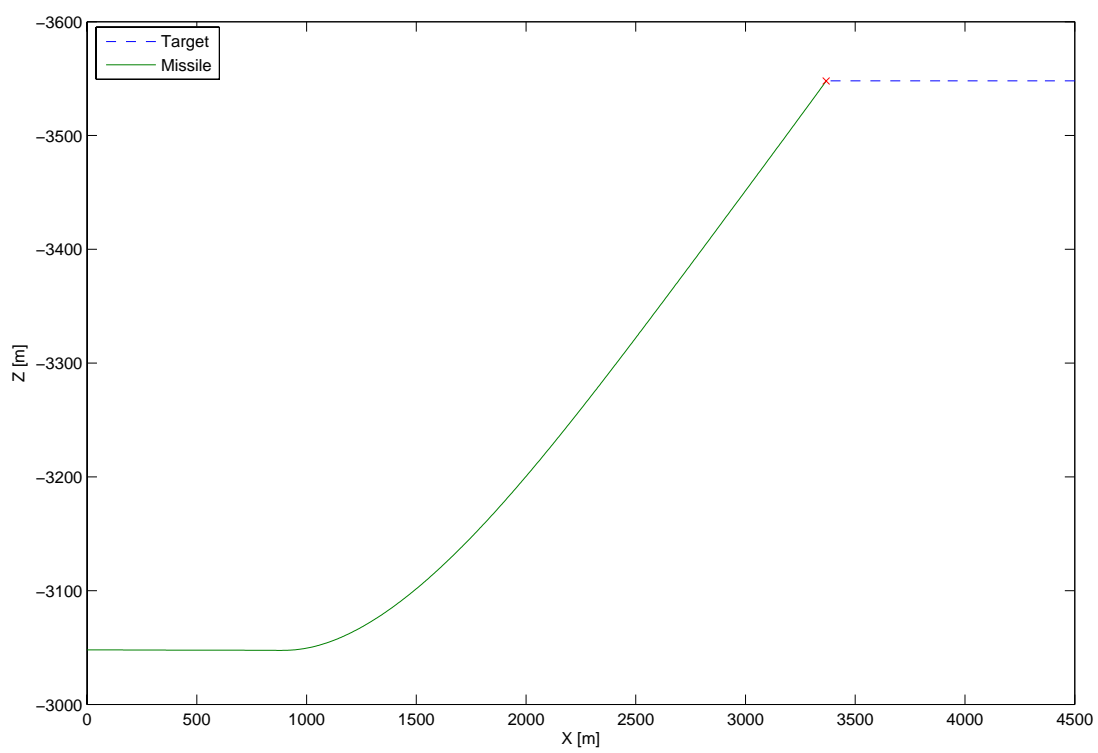

Figure 3. Interceptor and target trajectories for the nominal engagement scenario, no adaptive case. 

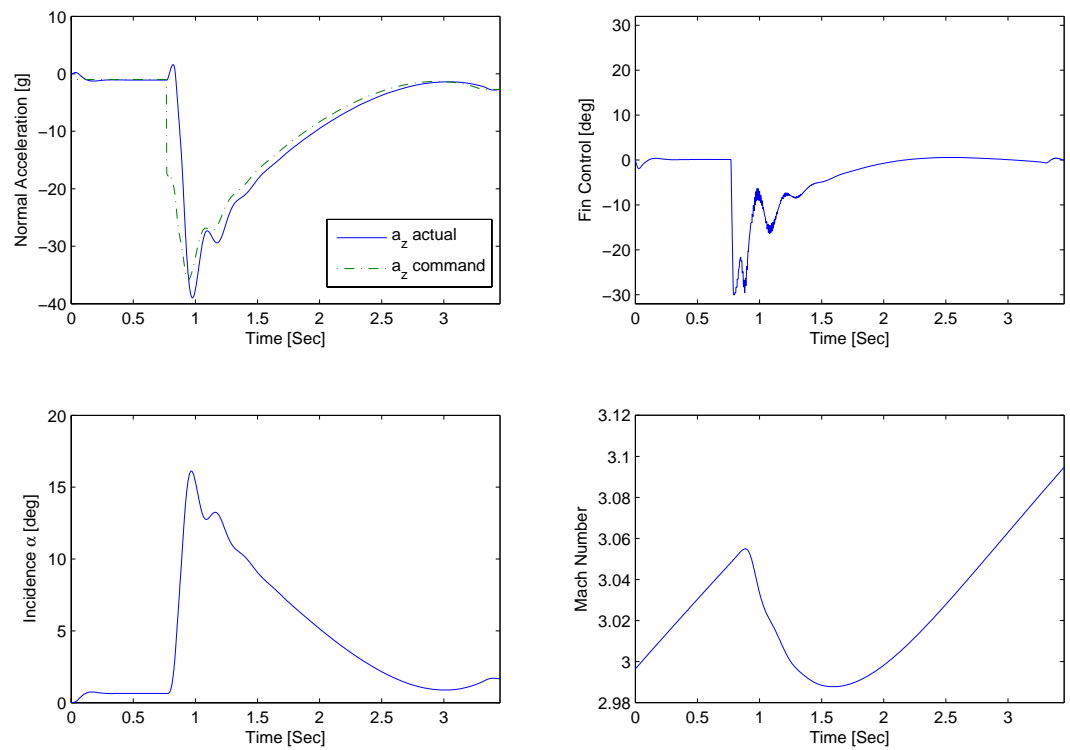

Figure 4. Time histories of measurements and control inputs for the nominal engagement scenario, augmented adaptive case.

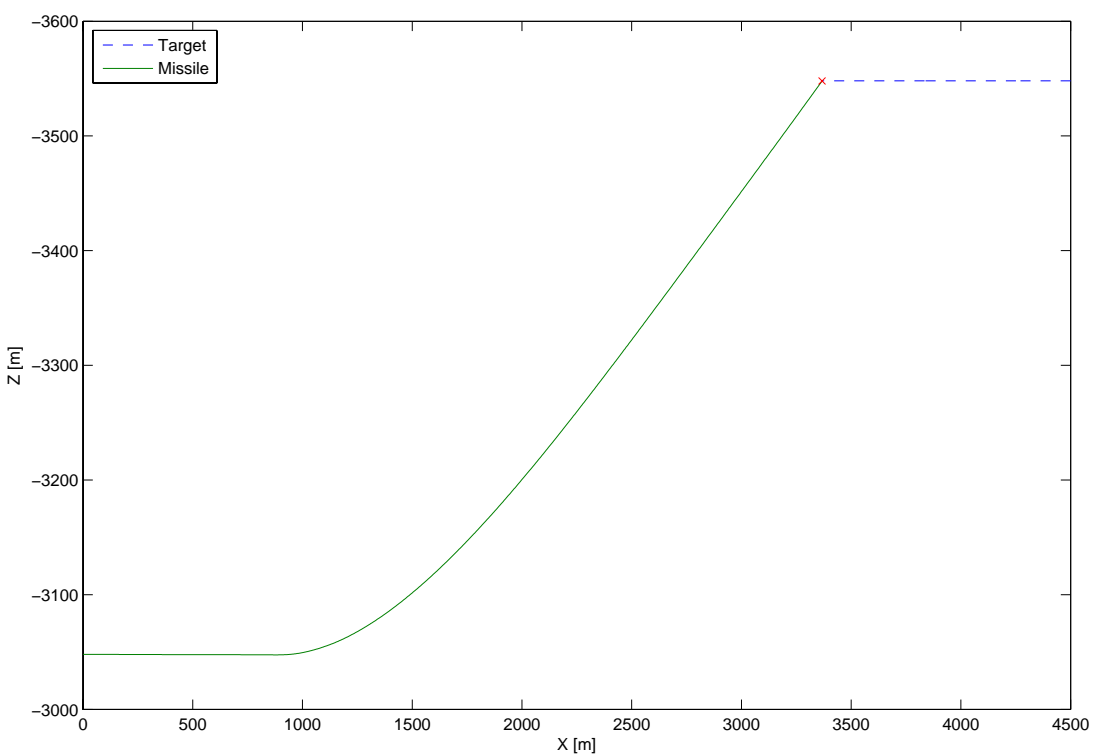

Figure 5. Interceptor and target trajectories for the nominal engagement scenario, augmented adaptive case. 

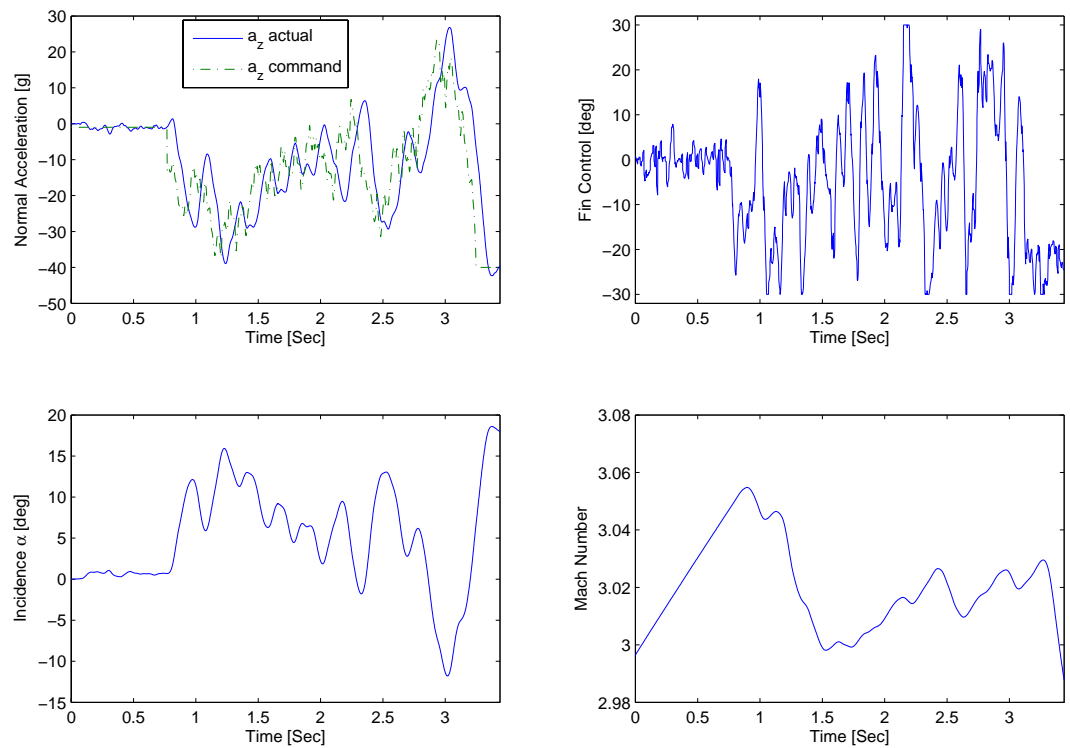

Figure 6. Time histories of measurements and control inputs for the applied rate gyro noise disturbance, no adaptive case.

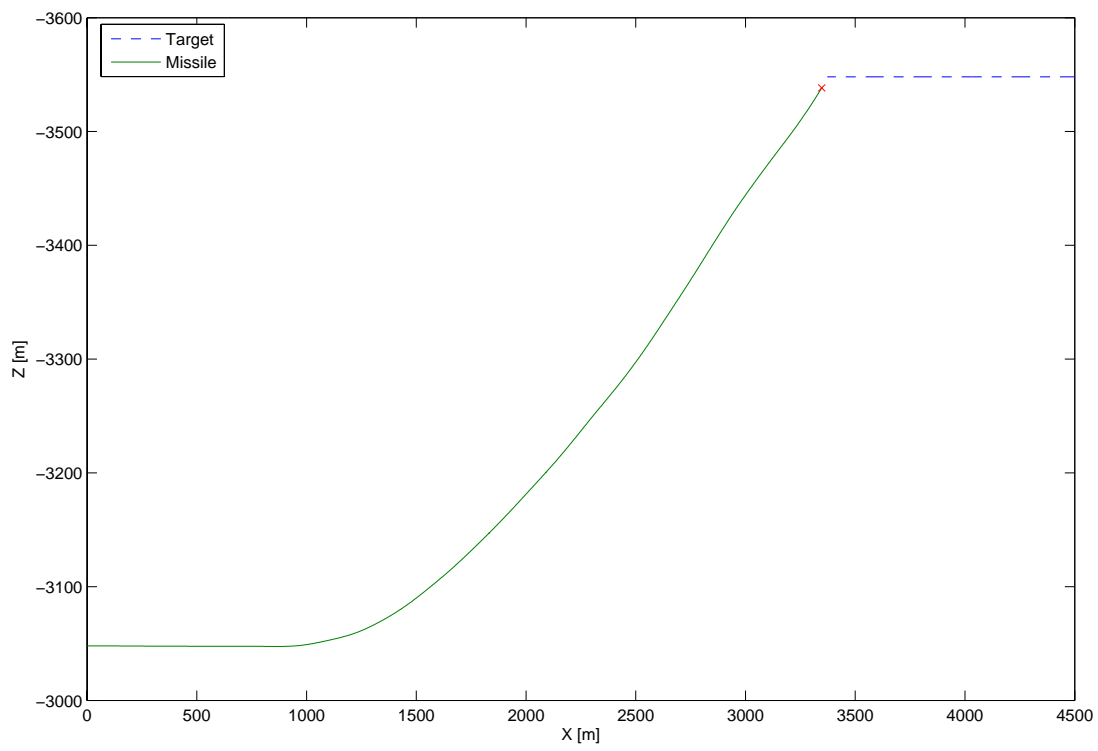

Figure 7. Interceptor and target trajectories for the applied rate gyro noise disturbance, no adaptive case. 

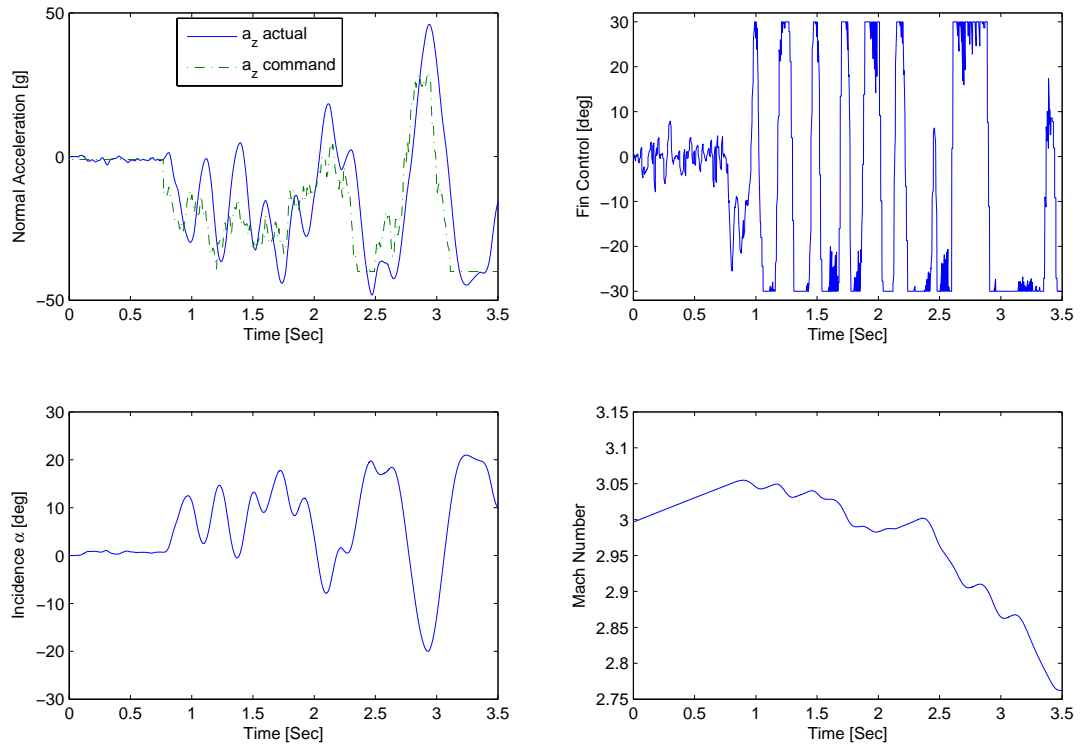

Figure 8. Time histories of measurements and control inputs for the applied rate gyro noise disturbance, augmented adaptive case.

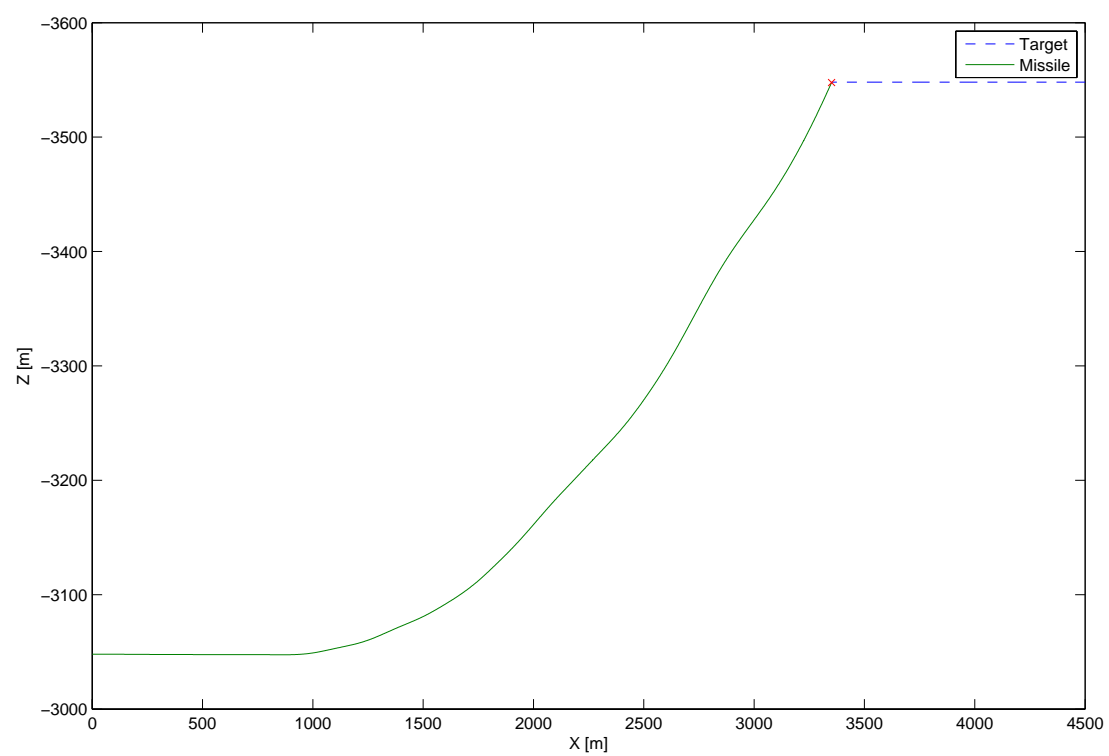

Figure 9. Interceptor and target trajectories for the applied rate gyro noise disturbance, augmented adaptive case. 

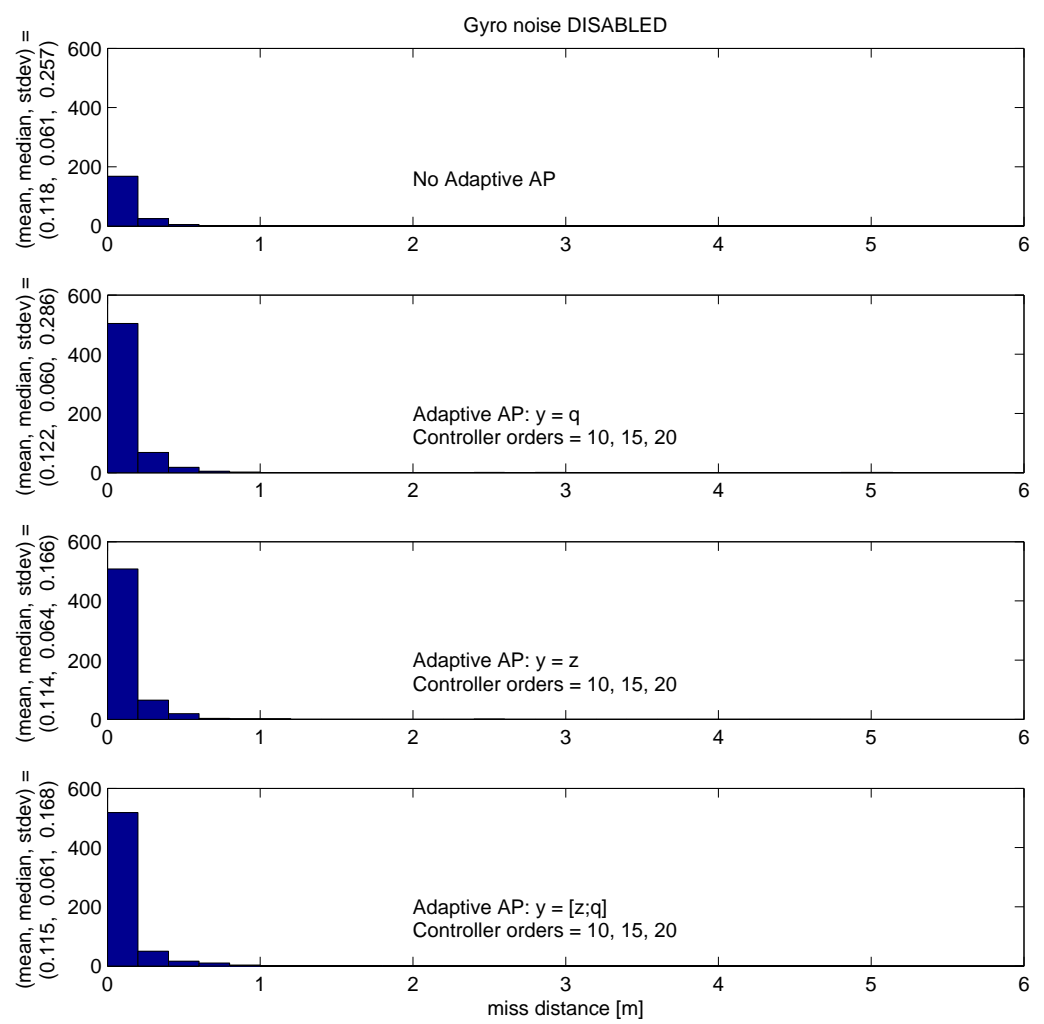

Figure 10. Miss distance histogram for different controller input combinations, no rate gyro noise disturbance, 2000 runs per design case.
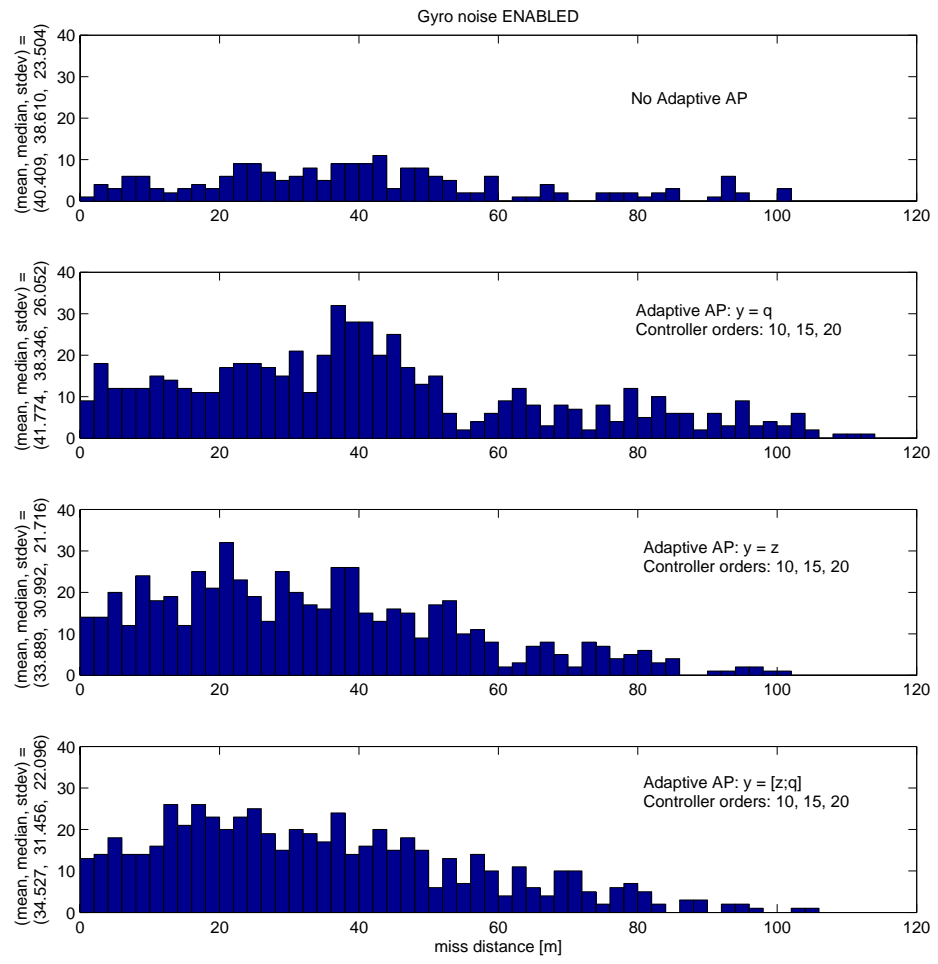

Figure 11. Miss distance histogram for different controller input combinations, rate gyro noise disturbance enanbled, 2000 runs per design case. 

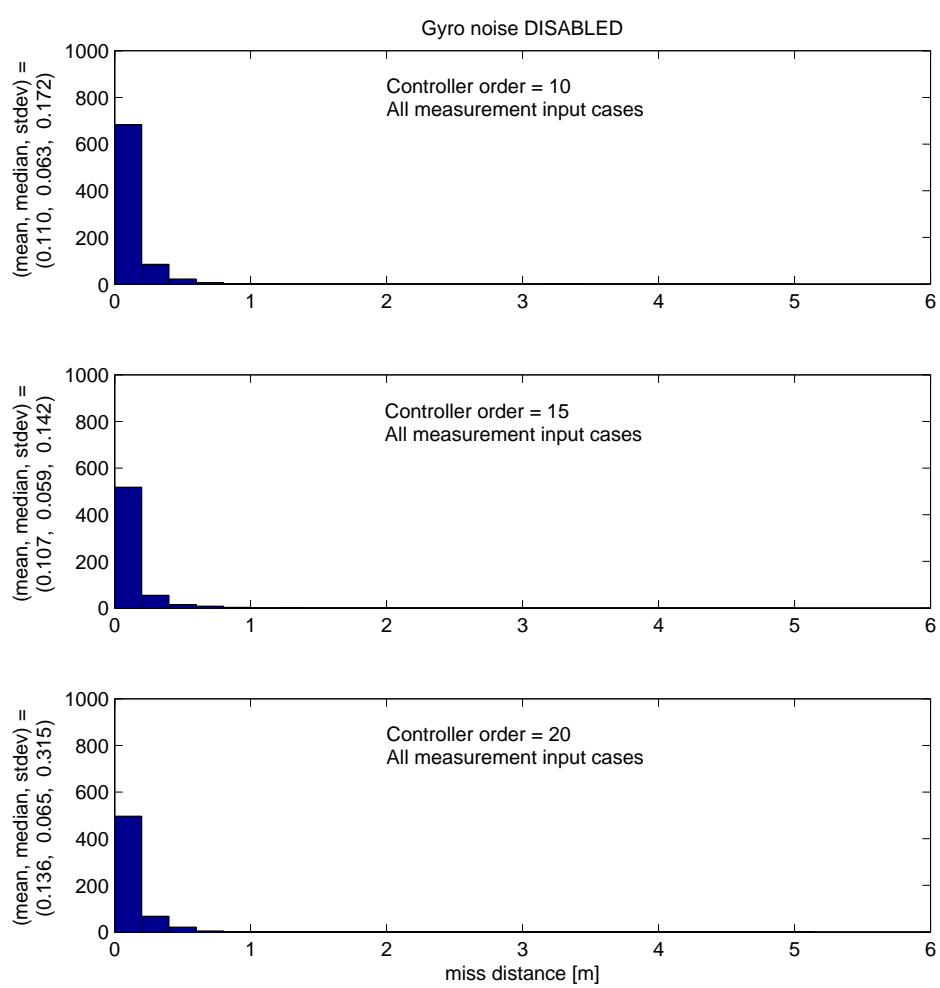

Figure 12. Miss distance histogram for different adaptive controller orders, no rate gyro noise disturbance, 2000 runs per design case.
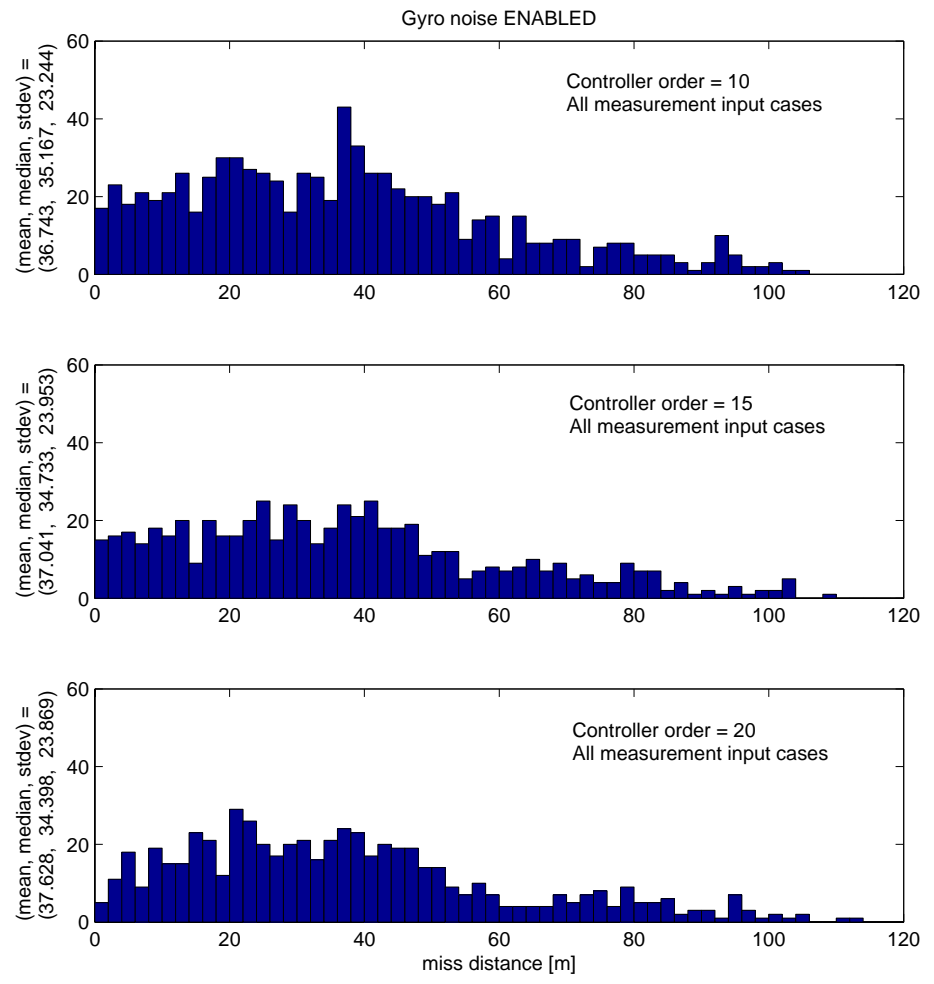

Figure 13. Miss distance histogram for different adaptive controller orders, rate gyro noise disturbance enabled, 2000 runs per design case. 


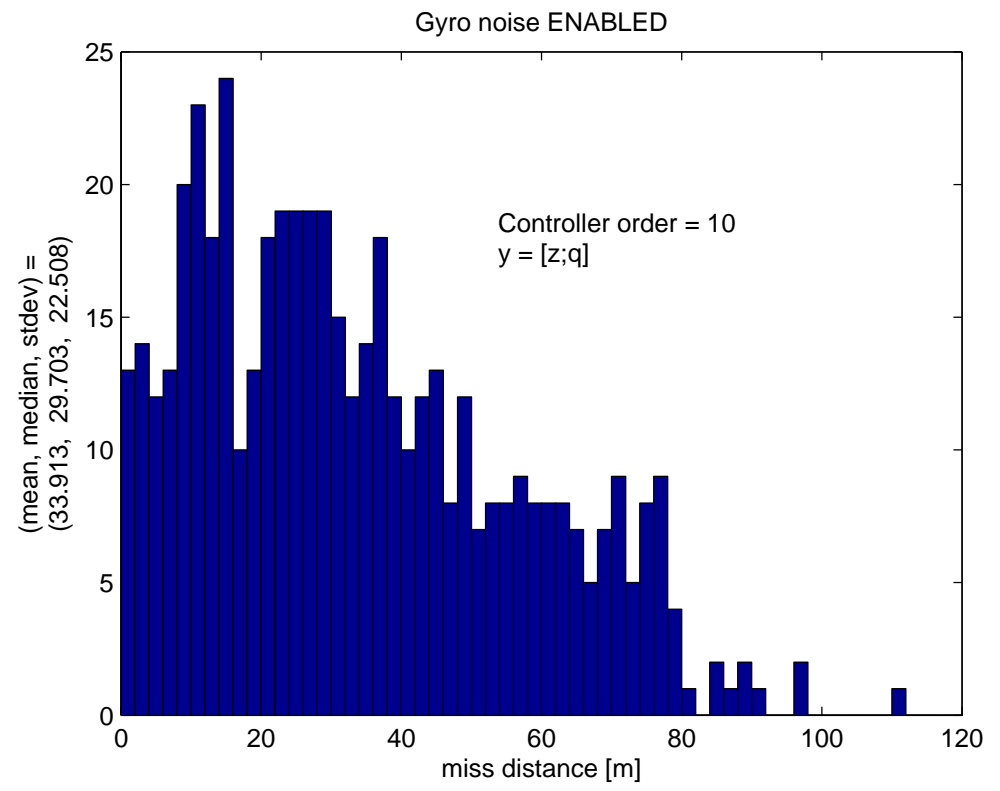

Figure 14. Miss distance histogram, $y=[z ; q]$, controller order $=\mathbf{1 0}$, rate gyro noise disturbance enabled, 2000 runs. 